\title{
Corona Mortis: Importancia Anatomo-Clínica y Ocurrencia en una Muestra de la Población Colombiana
}

\author{
Corona Mortis: Anatomical and Clinical Relevance and \\ Occurrence in a Sample of the Colombian Population
}

\author{
Gloria Baena*; Sebastián Rojas* \& Elizabeth Peña*
}

BAENA, G. ; ROJAS, S. \& PEÑA, E. Corona mortis: importancia anatomo - clínica y ocurrencia en una muestra de la población colombiana. Int. J. Morphol., 33(1):130-136, 2015.

RESUMEN: Corona mortis es una variación anatómica referida a la anastomosis de los vasos iliacos externos e internos, mediante vasos que se arquean por encima y por detrás del ramo superior del pubis. En caso de resultar lesionados iatrogénicamente, se pueden presentar hemorragias de difícil manejo. El objetivo del estudio fue caracterizar los vasos obturatrices en una muestra cadavérica de la población colombiana, para determinar su morfometría y la frecuencia con que se presentan los diferentes patrones venosos y arteriales. Se estudiaron hemipelvis de 14 cadáveres, pertenecientes al anfiteatro del Departamento de Morfología de la Universidad del Valle en Cali, Colombia. Tras acceder a la cavidad pélvica y completar la disección de todos los vasos que se relacionaban con el canal obturador y la cara pélvica del ramo superior del pubis, se determinaron sus características morfológicas y sus dimensiones. Las variaciones arteriales, corona mortis y arteria obturatriz aberrante, se presentaron en el 35,7\% de las hemipelvis y las variaciones venosas en el $82,1 \%$. La arteria corona mortis sólo se presentó en el 3,6\% de las hemipelvis y el 32,1\% presentaron arteria obturatriz aberrante. La moda de las frecuencias absolutas de los patrones venosos fue la vena corona mortis que se presentó en el 75,0\% de las hemipelvis. El conocimiento de los patrones arteriales y venosos de los vasos obturatrices y de sus variaciones anatómicas para la población colombiana es indispensable para evitar lesionarlos durante procedimientos clínico - quirúrgicos que comprometan el anillo pélvico anterior y la región inguinal.

PALABRAS CLAVE: Variación anatómica; Enfermedad iatrogénica; Sangrado; Hernia abdominal; Fracturas óseas; Arteria iliaca; Vena iliaca; Arterias epigástricas; Huesos de la pelvis; Pubis.

\section{INTRODUCCIÓN}

La arteria obturatriz suele originarse de la arteria iliaca interna, emerge por el canal obturador a la región femoral medial y pueden presentarse variados patrones de anastomosis entre ella y las arterias iliaca externa y epigástrica inferior. Igual patrón suele presentarse para las venas (Standring, 2005; Chatain \& Bustamante, 1986; Pró, 2012). Una de estas variaciones es la arteria obturatriz aberrante, la cual no se origina de la iliaca interna, sino de la arteria iliaca externa o de la epigástrica inferior y emerge a la región femoral por el canal obturador. El origen anómalo de esta arteria es lo que hace que se le considere arteria obturatriz aberrante (Darmanis et al., 2007; Sakthivelavan et al., 2010). Otra variación anatómica es la llamada arteria corona mortis, en cuyo caso la arteria obturatriz presenta su patrón normal de origen en el tronco anterior de la iliaca interna, pero existe una arteria adicional y de menor calibre, originada en la arteria iliaca externa o en la epigástrica infe- rior, que se arquea sobre el ramo superior del pubis, pasa por detrás de él y se anastomosa con la arteria obturatriz (Sakthivelavan et al.; Rusu et al., 2010; Berberoglu et al., 2001; Mahato 2009; Stavropoulou-Deli \& Anagnostopoulou, 2013; Smith et al., 2009). Teniendo en cuenta esta distinción conceptual, cabe anotar que ambas formas de variación suponen un riesgo en las intervenciones quirúrgicas que involucran la parte inferior de la pared abdominal anterior, ya que en ambas los vasos discurren por encima y por detrás del ramo superior del pubis.

El nombre de corona mortis se traduce del latín como corona mortuoria o corona de la muerte debido a que, en caso de que estos vasos sean accidentalmente lesionados, la hemorragia subsiguiente, altamente significativa y de difícil manejo, pone en riesgo la vida de la persona (Darmanis et al.; Stavropoulou-Deli \& Anagnostopoulou; Smith et al.; 
Bataduwaarachchi \& Thillainathan, 2011). Este evento y la posterior retracción de los vasos hacia la cavidad pélvica, pueden ocurrir en fracturas del pubis o durante intervenciones quirúrgicas que involucran la parte inferior de la pared abdominal anterior, tales como herniorrafias inguinales y femorales, cistectomías radicales, accesos anteriores al acetábulo como el ilioinguinal o el intrapélvico, procedimientos para el tratamiento de la incontinencia urinaria de esfuerzo como la TVT Secur y otras maniobras quirúrgicas que involucran la región retropúbica (Darmanis et al.; Mahato; Stavropoulou-Deli \& Anagnostopoulou; Smith et al.; Karakurt et al., 2002; Sarikcioglu \& Sindel, 2002). No obstante, la ventaja que ofrecen estas anastomosis es que se puede contar con una vía de circulación colateral cuando se presentan algunos tipos de obstrucción aortoiliaca (Jaburek et al., 2011).

Los patrones arteriales y venosos de los vasos obturatrices, así como sus variaciones, corona mortis y vasos obturatrices aberrantes, su morfometría y la frecuencia con que se presentan en una muestra cadavérica de la población colombiana, fueron el objetivo del presente estudio, el cual contribuirá a enriquecer el conocimiento sobre estos vasos y su aplicación clínica.

\section{MATERIAL Y MÉTODO}

Se realizó un estudio descriptivo, observacional de corte transversal, en el que se tomó como población de estudio las hemipelvis de 14 cadáveres, 3 femeninos y 11 masculinos, pertenecientes al anfiteatro del Departamento de Morfología de la Universidad del Valle, en Cali, Colombia. Se tuvieron como criterios de inclusión el que la pared abdominal anterolateral estuviera totalmente disecada para poder acceder más fácilmente a la cavidad pélvica desde su abertura superior, y que los vasos iliacos internos y externos estuvieran presentes. Se excluyeron los cadáveres en los cuales las ramas de estos vasos estaban seccionadas.
Se procedió a disecar totalmente todos los vasos que se relacionaban con el canal obturador y con el ramo superior del pubis, se determinó qué patrón de variación presentaban (Tabla I) y se midieron tanto la longitud como el calibre, empleando un calibrador digital electrónico marca ${ }^{\circledR}$ Bulltools, con resolución de $0,01 \mathrm{~mm} /$ 0,0005", precisión $\pm 0,02 \mathrm{~mm} / 0,001$ " y repetibilidad de $0,01 \mathrm{~mm} / 0,0005$ ". La lateralidad de la hemipelvis también fue registrada. Para la medida de longitud se midió el largo del vaso desde su origen hasta su terminación o hasta el canal obturador. Para determinar el calibre de los vasos, y considerando que ellos presentan un perímetro diferente a lo largo de su recorrido, se midieron éstos a nivel del origen del vaso, en su parte media y cerca de su terminación; arbitrariamente se denominaron estos puntos como tercios proximal, medio y distal, respectivamente. Cada perímetro fue dividido por una aproximación del número $\pi(3,1416)$ para obtener la medida del diámetro del vaso, y se tomó como diámetro del vaso el promedio de las tres mediciones. Con el fin de asegurar que los datos fueran confiables, con los investigadores participantes se realizaron pruebas interobservador para todas las mediciones.

Para el análisis de los datos se utilizó estadística descriptiva. Debido a que los diámetros de los vasos no tuvieron una distribución gaussiana, para todas las mediciones se determinaron el promedio, la desviación estándar y la mediana, en tanto que para las variables nominales se usaron frecuencias porcentuales.

\section{RESULTADOS}

Las variaciones arteriales, corona mortis y arteria obturatriz aberrante, se presentaron en el $35,7 \%$ de las hemipelvis y las variaciones venosas en el $82,1 \%$. La moda de las frecuencias absolutas de los patrones arteriales fue el patrón normal (17 hemipelvis) que corresponde al 60,7\%

Tabla I. Patrones de las variaciones de los vasos obturatrices.

\begin{tabular}{ll}
\hline Nombre del patrón & Descripción anatómica \\
\hline Vaso con patrón normal & $\begin{array}{l}\text { Vaso obturador que cumple con la definición de lo tradicionalmente descrito en la literatura como un } \\
\text { Vaso obturador. }\end{array}$ \\
& $\begin{array}{l}\text { Vaso que drena o p roviene del vaso iliaco externo o del vaso epigástrico inferior, se arquea por } \\
\text { encima y p or detrás del ramo superior del pubis, desciende y se anastomosa o n o con el vaso } \\
\text { obturador; si no se anastomosa, cursa junto con el vaso obturador a través del canal obturador. }\end{array}$ \\
Vaso obturatriz aberrante & $\begin{array}{l}\text { Vaso que drena o p roviene del vaso iliaco externo o del vaso epigástrico inferior, se arquea por } \\
\text { encima y por detrás del ramo superior del pubis, desciende en dirección del canal obturador y } \\
\text { reemplaza totalmente la irrigación que proveería el vaso obturador. } \\
\text { Otra variación }\end{array}$ \\
$\begin{array}{l}\text { Corresponde a un vaso obturador accesorio aberrante que se encuentra ubicado superior y paralelo al } \\
\text { vaso obturador, pero no se relaciona con la cara pélvica del ramo superior del pubis; por lo tanto, no } \\
\text { es objeto directo del presente estudio. }\end{array}$ \\
\hline
\end{tabular}




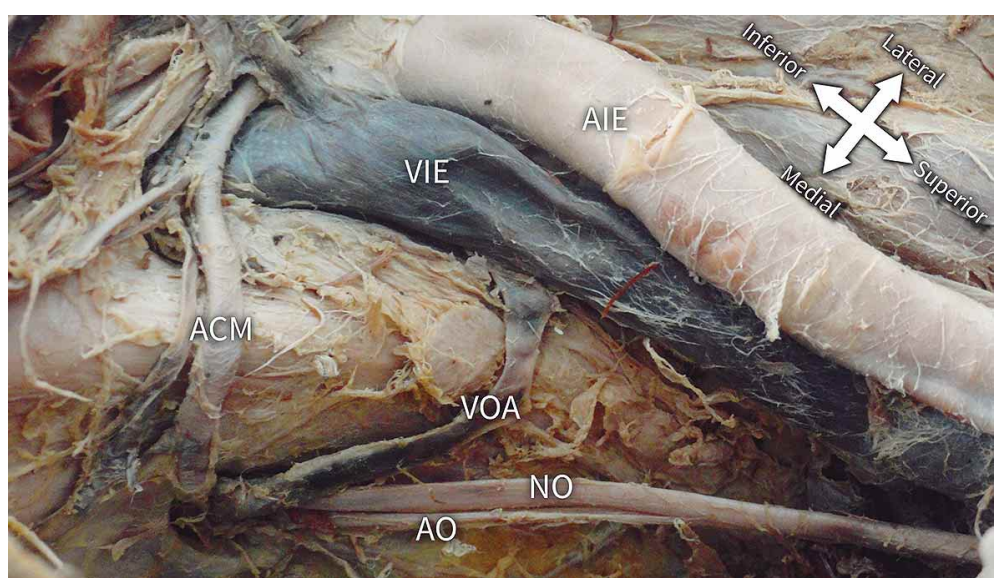

Fig. 1. Hemipelvis derecha con una arteria corona mortis (ACM) que proviene directamente de la arteria iliaca externa (AIE). Se observa la arteria obturatriz (AO) alcanzando el canal obturador derecho inmediatamente por debajo del nervio obturador $(\mathrm{NO})$. Adicionalmente, se encuentra una vena obturatriz aberrante (VOA), tributaria de la vena iliaca externa (VIE).

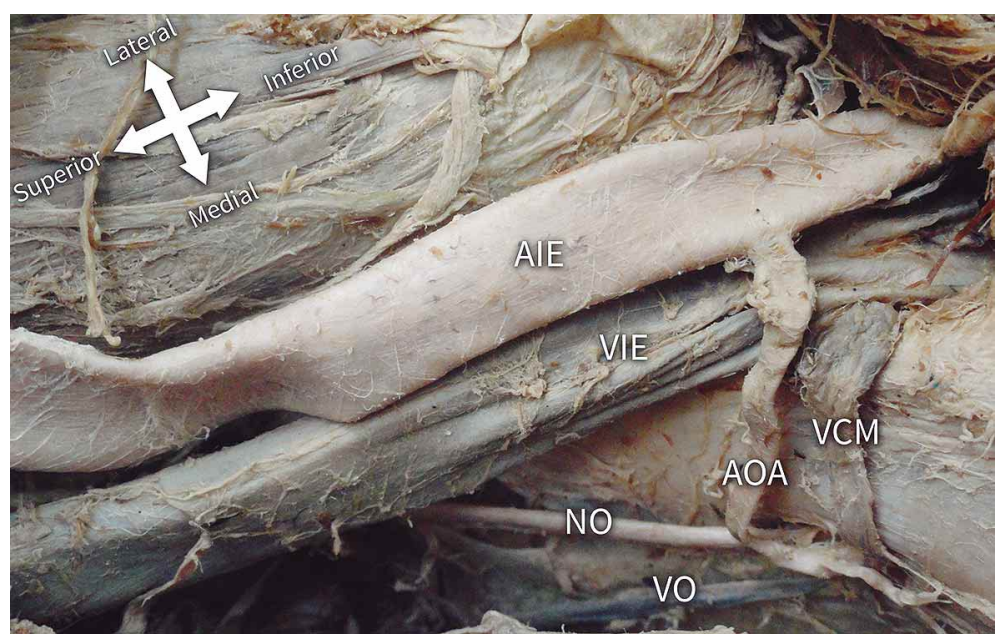

Fig. 2. Hemipelvis izquierda con una arteria obturatriz aberrante (AOA) que proviene de la arteria iliaca externa (AIE). Adicionalmente, se encuentra una vena corona mortis (VCM) tributaria de la vena iliaca externa (VIE) y la vena obturatriz (VO) que cursa por debajo del nervio obturador (NO).

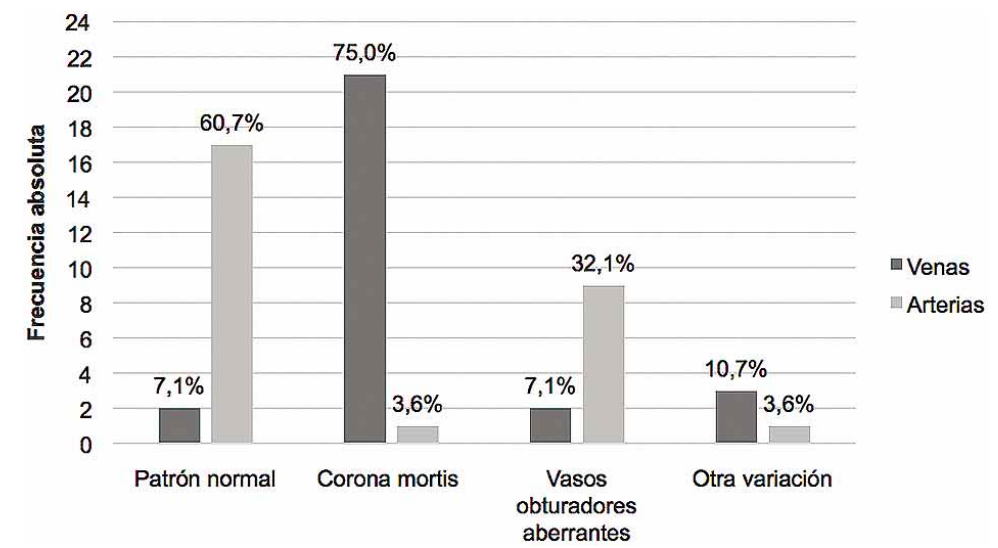

Fig. 3. Frecuencias absolutas y porcentuales de las variaciones arteriales y venosas de los vasos obturatrices según el patrón de variación. de la muestra. La arteria corona mortis sólo se presentó en una hemipelvis $(3,6 \%)$ (Fig. 1) y 9 presentaron arteria obturatriz aberrante $(32,1 \%)$ (Figs 2 y 3).

La Moda de las frecuencias absolutas de los patrones venosos fue la vena corona mortis (21 hemipelvis), que corresponde al $75,0 \%$ de las hemipelvis (Fig. 2), con una frecuencia absoluta para los patrones normal y vena obturatriz aberrante de 2 cada uno (Fig. 1), lo cual representa el $7,1 \%$ de la muestra, respectivamente (Fig. 3).

El promedio de las longitudes de las arterias que guardaban relación con la cara pélvica del ramo superior del pubis $(4,4 \mathrm{~cm}$ para la arteria corona mortis; 4,9 $\pm 0,873 \mathrm{~cm}$ para la arteria obturatriz aberrante) fue similar al presentado por las venas con la misma relación anatómica y el mismo patrón de variación $(4,2 \pm 0,878 \mathrm{~cm}$ para la vena corona mortis; $5,0 \pm 0,212 \mathrm{~cm}$ para la vena obturatriz aberrante) (Fig. 4). Por otra parte, la mediana de los diámetros de los vasos obturatrices aberrantes (0,3 cm para las venas y las arterias) fue mayor que la de los vasos corona mortis $(0,2 \mathrm{~cm}$ para las venas y las arterias) (Fig. 5).

\section{DISCUSIÓN}

Dado que la prevalencia estimada de herniorrafias inguino-femorales fluctúa entre el 10 y el $15 \%$ para la población general (Pol Herrea et al., 2011), se justifica realizar estudios que lleven a profundizar en el conocimiento de las estructuras anatómicas involucradas en dichas intervenciones. Aunque de baja ocurrencia, la inestabilidad hemodinámica que puede acompañar una fractura del ramo superior del pubis, aparentemente poco complicada, puede ser causada por la lesión de un vaso sanguíneo que curse por detrás de éste (Calero et al., 2012).

La corona mortis se describe clásicamente como una variación vascular de tipo arterial, lo que conlleva a que se encuentren menos reportes sobre las variaciones venosas que sobre las arteriales, dejando de lado su importancia clínica al tener la misma disposi- 


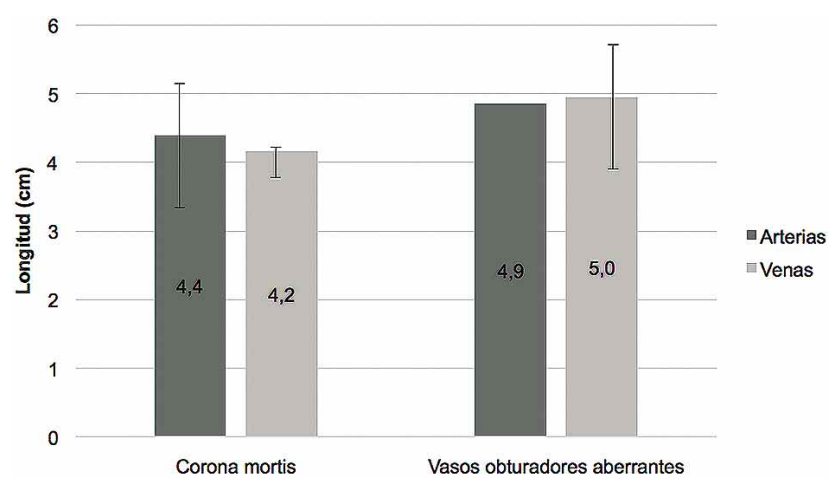

Fig. 4. Promedios de las longitudes de los vasos que se relacionan con la cara pélvica del ramo superior del pubis, según el patrón de la variación.

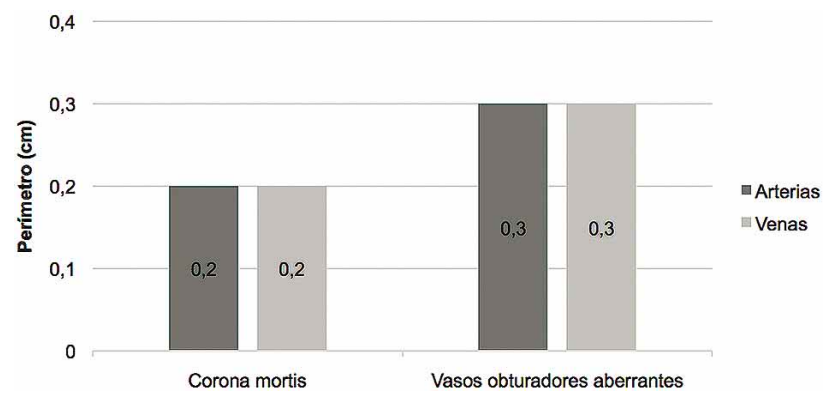

Fig. 5. Medianas de los diámetros de los vasos relacionados con la cara pélvica del ramo superior del pubis, según el patrón de la variación.

ción morfológica y la misma posibilidad de sangrado iatrogénico (Sakthivelavan et al.; Sarikcioglu et al., 2003; Namking et al., 2007). Darmanis et al. plantean la paradoja de la baja incidencia de sangrado iatrogénico secundario a la lesión de un vaso que curse por la cara pélvica del ramo superior del pubis, aunque la prevalencia de la variación en las disecciones de cadáveres sea considerable; incluso exponen varias teorías que explican el fenómeno, entre ellas que el vaso lesionado sufra vasoespasmo por estrés (Darmanis et al.). Nosotros postulamos otra posible explicación, donde al tener mayor ocurrencia la variación venosa, el vaso lesionado sea una vena y, dado que la presión sanguínea en las venas es más baja y éstas tienden a colapsarse, el sangrado no resultaría significativo en caso de ruptura.

Darmanis et al. y Namking et al. encontraron que no hay diferencias significativas en cuanto al sexo en las variables analizadas sobre los vasos obturatrices y sus variaciones. En el presente estudio no se consideró analizar esta relación debido a los antecedentes para otras poblaciones y a la baja ocurrencia de cadáveres femeninos de la muestra.
En el actual estudio se encontró que 35,7\% de los vasos arteriales y $82,1 \%$ de los vasos venosos cursaban por la cara pélvica del ramo superior del pubis, datos que se asemejan a los del estudio de Tornetta et al. (1996), realizado en Estados Unidos y citado por Rusu et al. (2010)., con una población de estudio de 50 cadáveres.

En la revisión de la literatura realizada por Darmanis et al., se reporta que la arteria obturatriz aberrante tiene un rango de prevalencia del 20 al 30\%, valor cercano al encontrado para la población colombiana del presente estudio $(32,1 \%)$. Contrariamente, se reporta que el rango de prevalencia de la arteria corona mortis es del 10 al $43 \%$, rango substancialmente mayor al del presente estudio $(3,6 \%)$. En la literatura se reporta mayor prevalencia de corona mortis que de arteria obturatriz aberrante, lo cual es contrario a lo encontrado para la población colombiana estudiada. Para las venas la situación es diferente, encontrándose que la vena corona mortis se presenta en el 75,0\% de las hemipelvis, valor que se encuentra dentro del rango reportado para la población general (52 al 96\%). Este rango tan amplio y las diferencias de las frecuencias encontradas en muchos estudios, sugiere lo impredecible de la ocurrencia de las variaciones venosas (Mahato).

Dado que solamente 2 hemipelvis $(7,1 \%)$ presentaron el patrón de normalidad venoso reportado en la literatura, 92,9\% de las hemipelvis no se ajustaron a él, y de este porcentaje el $75 \%$ correspondió a la vena corona mortis. Lo anterior permite concluir que, para la muestra de población colombiana estudiada, el patrón venoso que puede considerarse normal es uno de los considerados como variación para otras poblaciones; es decir, el patrón de la vena obturatriz que desemboca en la vena iliaca interna, pero que también existe una vena que cursa por detrás del ramo superior del pubis y desemboca en la vena iliaca externa o en la vena epigástrica inferior. Vale la pena corroborar este patrón venoso de la población colombiana con muestras de mayor tamaño y de diferente procedencia regional. Por el contrario, en la mayoría de las arterias $(60,7 \%)$ se conservó el patrón de normalidad descrito clásicamente. Sin embargo, no se puede perder de vista la cantidad considerable de hemipelvis $(39,3 \%)$ que presentan variaciones. Por la baja prevalencia de corona mortis arterial encontrada para la población del estudio $(3,6 \%)$ y la tan alta prevalencia de corona mortis venosa (75\%), no se puede descartar la posibilidad de que la población estudiada no refleje totalmente la realidad de la población colombiana, lo cual implica la necesidad de realizar estudios a mayor escala.

La Tabla II compara el estudio actual con estudios 
Tabla II. Frecuencias porcentuales de las variaciones de los vasos obturatrices reportadas por otros autores

\begin{tabular}{|c|c|c|c|c|}
\hline Autores, año y país de realización & $\begin{array}{c}\text { Vasos que } \\
\text { cruzan por } \\
\text { el ramo } \\
\text { superior } \\
\text { del pubis } \\
(\%) \\
\end{array}$ & $\begin{array}{c}\text { Arterias que } \\
\text { cruzan por el } \\
\text { ramo } \\
\text { superior del } \\
\text { pubis }(\%)\end{array}$ & $\begin{array}{c}\text { Venas que } \\
\text { cruzan por el } \\
\text { ramo } \\
\text { superior del } \\
\text { pubis }(\%)\end{array}$ & Población de estudio \\
\hline Missankov et al. (1996) - Sudáfrica & --- & --- & 46 & 49 hemipelvis \\
\hline Tornetta et al. (1996) - Estados Unidos & 84 & 34 & 70 & 50 hemipelvis \\
\hline de Kleuver et al. (1998) -Países Bajos & 25 & --- & --- & 24 hemipelvis \\
\hline Berberoglu et al. (2001) - Turquía & --- & --- & 100 & 14 hemipelvis \\
\hline Karakurt et al. (2002) - Turquía & --- & 28,5 & --- & 98 angiografías pacientes \\
\hline Sarikcioglu et a l. (2003) - Turquía & --- & 0 & 20,37 & 54 hemic adáveres \\
\hline Hong et al. (2004) - China & 72 & --- & --- & 50 hemipelvis \\
\hline Okcu et al. (2004) - Turquía & 61 & 19 & 52 & 150 hemicadáveres \\
\hline Pungpapong \& Thum-umnauysuk (2005) - Tailandia & 77,27 & --- & --- & 66 hemipelvis \\
\hline Darmanis et al. (2007) - Reino Unido & 83 & --- & --- & 80 hemipelvis \\
\hline Rusu et al. (2010 - Rumania & 80 & 65 & 55 & 40 hemipelvis \\
\hline Estu dio actual (2015) - Colombia & 82,1 & 35,7 & 82,1 & 28 hemipelvis \\
\hline
\end{tabular}

previos que reportan las frecuencias porcentuales de la ocurrencia de vasos que se relacionan con la cara pélvica del ramo superior del pubis. Se observa que en el estudio actual, el porcentaje de hemipelvis que presentaron algún vaso relacionado con la cara pélvica del ramo superior del pubis, es igual al porcentaje de hemipelvis con venas que presentaban esta misma relación, pero no ocurre igual para las arterias; es decir, que al presentarse este patrón podía no estar presente una arteria pero siempre estaba presente una vena.

En el actual estudio se encontró que las medidas de la longitud y el diámetro de los vasos obturatrices aberrantes son mayores que las de los vasos corona mortis, y esto concuerda con la descripción hecha por Darmanis et al., de que el vaso obturatriz aberrante es un vaso "más grande" (Darmanis et al.). De lo anterior puede inferirse que si se lesionan los vasos obturatrices aberrantes, el sangrado va a ser más profuso que si se lesionan los vasos corona mortis.
En la literatura revisada no se encontraron estudios que reporten la longitud de los vasos, pero sí se encontraron varios con las medidas del diámetro de los vasos corona mortis. La Tabla III resume los datos encontrados.

Es relevante conocer las posibles variaciones que pueden presentar los vasos obturatrices en la población colombiana para evitar lesionarlos en procedimientos clínico - quirúrgicos donde estén implicados el anillo pélvico anterior y la región inguinal.

\section{AGRADECIMIENTOS}

Los autores agradecen al Departamento de Morfología de la Universidad del Valle el permitir el uso del anfiteatro para el desarrollo del presente estudio.

Tabla III. Diámetros de los vasos obturatrices reportados por otros autores.

\begin{tabular}{|c|c|c|c|c|c|}
\hline Autores, año y país de realización & $\begin{array}{c}\text { Diámetro } \\
\text { de los vasos } \\
\text { corona } \\
\text { mortis } \\
(\mathrm{mm})\end{array}$ & $\begin{array}{c}\text { Diámetro } \\
\text { de las } \\
\text { arterias } \\
\text { corona } \\
\text { mortis } \\
(\mathrm{mm})\end{array}$ & $\begin{array}{c}\text { Diámetro } \\
\text { de las } \\
\text { venas } \\
\text { corona } \\
\text { mortis } \\
(\mathrm{mm})\end{array}$ & $\begin{array}{c}\text { Diámetro } \\
\text { de las } \\
\text { arterias } \\
\text { obturatrices } \\
\text { aberrantes } \\
(\mathbf{m m})\end{array}$ & $\begin{array}{c}\text { Población de } \\
\text { estudio }\end{array}$ \\
\hline B erberoglu et al. (2001) - Turquía & --- & 0,98 & 3,3 & 2,6 & 14 hemipelvis \\
\hline Okcu et al. (2004) - Turquía & 2 & --- & --- & --- & 150 hemicadáveres \\
\hline Darmanis et al. (2007) - Reino Unido & 2,6 & --- & --- & --- & 80 hemipelvis \\
\hline Rusu et al. (2010) - Rumania & $2-4,2$ & --- & --- & --- & 40 hemipelvis \\
\hline B ataduwaarachchi \& Thillainathan (2011) - Sri Lanka & 2,5 & 2 & 3 & --- & 1 cadáver \\
\hline Stavropoulou-Deli \& Anagnostopoulou (2013) - Grecia & 3,1 & 3 & 3,1 & --- & 70 hemicadáveres \\
\hline Estudio actual (2015) - Colombia & 2 & 2 & 2 & 3 & 28 hemipelvis \\
\hline
\end{tabular}


BAENA, G.; ROJAS, S. \& PEÑA, E. Corona mortis: Anatomical and clinical relevance and occurrence in a sample of the Colombian population. Int. J. Morphol., 33(1):130-136, 2015.

SUMMARY: The corona mortis is an anatomical variation related to the anastomosis between the internal and external iliac blood vessels, through certain arteries and veins that form arches above and behind the superior pubic ramous. Serious hemorrhages can result from iatrogenic injury of these vessels. The study objective was to characterize the obturator vessels in a sample of Colombian cadavers, to determine their morphometric features and the frequencies in which the arterial and venous patterns are present. Hemipelvises of 14 cadavers from the dissection room of the Department of Morphology at the Universidad del Valle, in Cali, Colombia, were taken as sample. Once the dissection of the vessels related with the obturator canal and the pelvic surface of the superior pubic ramous was completed, their morphological features were determined and measurements were taken of their diameters and lengths. The arterial variations, corona mortis and aberrant obturator artery, were present in $35.7 \%$ of the hemipelvises and the venous variations in $82.1 \%$. The corona mortis artery was only present in $3.6 \%$ of the hemipelvises and $32.1 \%$ presented an aberrant obturator artery. The mode of the absolute frequencies of the venous patterns was the corona mortis vein, present in $75.0 \%$ of the hemipelvises. The knowledge of the arterial and venous patterns of the obturator vessels and their anatomical variations for the Colombian population is very important in order to avoid causing any injury to them in the course of clinical and surgical procedures that have to do with the anterior pelvic ring and the inguinal region.

KEY WORDS: Anatomic variation; Iatrogenic disease; Bleeding; Abdominal hernia; Bone fractures; Iliac artery; Iliac vein; Epigastric arteries; Pelvic bones; Pubic bone.

\section{REFERENCIAS BIBLIOGRÁFICAS}

Bataduwaarachchi, V. R. \& Thillainathan, S. Bilateral indirect inguinal hernia with bilateral corona mortis: A case study from a laparoscopic perspective. Int. J. Anat. Var., 4:152-4, 2011.

Berberoglu, M.; Uz, A.; Ozmen, M. M.; Bozkurt, M. C.; Erkuran, C.; Taner, S.; Tekin, A. \& Tekdemir, I. Corona mortis: an anatomic study in seven cadavers and an endoscopic study in 28 patients. Surg. Endosc., 15(1):72-5, 2001.

Calero, C. A.; Mifsut Miedes, D. \& Gomar Sancho, F. Inestabilidad hemodinámica e hidronefrosis bilateral por hemorragia intrapélvica masiva secundaria a fractura no desplazada de rama púbica. Rev. Esp. Cir. Osteoartic., 47(251):143-6, 2012.

Chatain, I. \& Bustamante, J. Anatomía Macroscópica Funcional y Clínica. Bogotá, Addison-Wesley Iberoamericana, 1986.

Darmanis, S.; Lewis, A.; Mansoor, A. \& Bircher, M. Corona mortis: an anatomical study with clinical implications in approaches to the pelvis and acetabulum. Clin. Anat., 20(4):433-9, 2007.

de Kleuver, M.; Kooijman, M. A.; Kauer, J. M. \& Veth, R. P. Pelvic osteotomies: anatomic pitfalls at the pubic bone. A cadaver study. Arch. Orthop. Trauma Surg., 117(4-5):270-2, 1998.

Hong, H. X.; Pan, Z. J.; Chen, X. \& Huang, Z. J. An anatomical study of corona mortis and its clinical significance. Chin. J. Traumatol., 7(3):165-9, 2004.

Jaburek, L.; Jaburkova, J.; Lubusky, M. \& Prochazka, M. Risk of haemorrhagic complications of retropubic surgery in females: anatomic remarks. Biomed. Pap. Med. Fac. Univ. Palacky Olomouc Czech Repub., 155(1):75-7, 2011.
Karakurt, L.; Karaca, I.; Yilmaz, E.; Burma, O. \& Serin, E. Corona mortis: incidence and location. Arch. Orthop. Trauma Surg., 122(3):163-4, 2002.

Mahato, N. K. Retro-pubic vascular anomalies: A study of abnormal obturator vessels. Eur. J. Anat., 13(3):121-6, 2009.

Missankov, A. A.; Asvat, R. \& Maoba, K. I. Variations of the pubic vascular anastomoses in black South Africans. Acta Anat. (Basel), 155(3):212-4, 1996.

Namking, M.; Woraputtaporn, W.; Buranarugsa, M.; Kerdkoonchorn, M. \& Chaijarookhanarak, W. Variation in Origin of the Obturator Artery and Corona mortis in Thai. Siriraj Med. J., 59(1):12-5, 2007.

Okcu, G.; Erkan, S.; Yercan, H. S. \& Ozic, U. The incidence and location of corona mortis: a study on 75 cadavers. Acta Orthop. Scand., 75(1):53-5, 2004.

Pol Herrea, P. G.; López Rodríguez, P. R.; León González, O.; Caiñas Román, J.; Cruz García, N.; Pando Santos, A. \& Rodríguez Galván, H. Cirugía de la hernia inguinal en la tercera edad: ambulatoria y con hospitalización corta. Rev. Cubana Cir., 50(1):73-81, 2011.

Pró, E. A. Anatomía Clínica. Buenos Aires, Médica Panamericana, 2012.

Pungpapong, S. U. \& Thum-umnauysuk, S. Incidence of corona mortis; preperitoneal anatomy for laparoscopic hernia repair. J. Med. Assoc. Thail., 88(Suppl. 4):S51-3, 2005. 
BAENA, G. ; ROJAS, S. \& PEÑA, E. Corona mortis: importancia anatomo - clínica y ocurrencia en una muestra de la población colombiana. Int. J. Morphol., 33(1):130-136, 2015.

Rusu, M. C.; Cergan, R.; Motoc, A. G.; Folescu, R. \& Pop, E. Anatomical considerations on the corona mortis. Surg. Radiol. Anat., 32(1):17-24, 2010.

Sakthivelavan, S.; Sendiladibban, S. D.; Aristotle, S. \& Sivanandan, A. V. Corona mortis - a case report with surgical implications. Int. J. Anat. Var., 3:103-5, 2010.

Sarikcioglu, L. \& Sindel, M. Multiple vessel variations in the retropubic region. Folia Morphol. (Warsz.), 61(1):435,2002 .

Sarikcioglu, L.; Sindel, M.; Akyildiz, F. \& Gur, S. Anastomotic vessels in the retropubic region: corona mortis. Folia Morphol. (Warsz.), 62(3):179-82, 2003.

Smith, J. C.; Gregorius, J. C.; Breazeale, B. H. \& Watkins, G. E. The corona mortis, a frequent vascular variant susceptible to blunt pelvic trauma: identification at routine multidetector CT. J. Vasc. Interv. Radiol., 20(4):455-60, 2009.

Standring, S. Gray's Anatomy: The Anatomical Basis of Clinical Practice. 39th ed. London, Elsevier Churchill Livingstone, 2005.

Stavropoulou-Deli, A. \& Anagnostopoulou, S. Corona mortis: anatomical data and clinical considerations. Aust. N. Z. J. Obstet. Gynaecol., 53(3):283-6, 2013.

Tornetta, P. 3rd.; Hochwald, N. \& Levine, R. Corona mortis. Incidence and location. Clin. Orthop. Relat. Res., (329):97-101, 1996.

\author{
Dirección para Correspondencia \\ Gloria Patricia Baena Caldas \\ Departamento de Morfología \\ Facultad de Salud \\ Universidad del Valle \\ Cali \\ COLOMBIA
}

Email: gloria.baena@correounivalle.edu.co

Recibido: 08-10-2014

Aceptado: 30-12-2014 